\title{
Two-Step MIR Inequalities for Mixed Integer Programs
}

\author{
Sanjeeb Dash \\ IBM T. J. Watson Research Center, Yorktown Heights, New York 10598, sanjeebd@us.ibm.com \\ Marcos Goycoolea \\ School of Business, Universidad Adolfo Ibáñez, Peñalolén, 7941169 Santiago, Chile, \\ marcos.goycoolea@uai.cl \\ Oktay Günlük \\ IBM T. J. Watson Research Center, Yorktown Heights, New York 10598, oktay@watson.ibm.com
}

\begin{abstract}
$\mathrm{T}_{\mathrm{m}}^{\mathrm{w}}$ wo-step mixed integer rounding (MIR) inequalities are valid inequalities derived from a facet of a simple mixed integer set with three variables and one constraint. In this paper we investigate how to effectively use these inequalities as cutting planes for general mixed integer problems. We study the separation problem for single-constraint sets and show that it can be solved in polynomial time when the resulting inequality is required to be sufficiently different from the associated MIR inequalities. We discuss computational issues and present numerical results based on a number of data sets.
\end{abstract}

Key words: mixed integer rounding; GMI cut; two-step MIR; group polyhedron; separation History: Accepted by Karen Aardal, Area Editor for Design and Analysis of Algorithms; received October 2007; revised June 2008; accepted March 2009. Published online in Articles in Advance.

\section{Introduction}

A useful technique in solving mixed integer programs is to extract a single implied constraint from the formulation of the problem and derive valid inequalities based on this constraint. Let

$$
P=\left\{v \in \mathbb{R}^{|J|}, x \in \mathbb{Z}^{|I|}: C v+A x=d, v, x \geq 0\right\},
$$

where the matrices $C$ and $A$ are of appropriate dimension. Let

$W=\left\{v \in \mathbb{R}^{|J|}, x \in \mathbb{Z}^{|I|}: \sum_{j \in J} c_{j} v_{j}+\sum_{i \in I} a_{i} x_{i} \geq b, v, x \geq 0\right\}$

be a relaxation of $P$, where the equation defining $W$, called the base inequality, is obtained by relaxing a linear combination of the equations defining $P$. Valid inequalities for $W$ can be used as cutting planes for $P$.

A well-known valid inequality for $W$ is the mixed integer rounding (MIR) inequality

$$
\sum_{j \in J} \max \left\{c_{j}, 0\right\} v_{j}+\sum_{i \in I}\left(\hat{b}\left\lfloor a_{i}\right\rfloor+\min \left\{\hat{b}, \hat{a}_{i}\right\}\right) x_{i} \geq \hat{b}\lceil b\rceil,
$$

where $\hat{b}=b-\lfloor b\rfloor$ and $\hat{a}_{i}=a_{i}-\left\lfloor a_{i}\right\rfloor$. The MIR inequality can be derived by mapping a point in $W$ to a point (via aggregation of variables) in

$$
Q^{1}=\{v \in \mathbb{R}, z \in \mathbb{Z}: v+z \geq \beta, v \geq 0\}
$$

and then using the only nontrivial facet of $Q^{1}$; see Wolsey (1998). As shown by Marchand and Wolsey (2001), when the base inequality is obtained from a row of a simplex tableau associated with $P$, the resulting inequality (2) is equivalent to the well-known Gomory mixed integer (GMI) cut.

Dash and Günlük (2006a) derived a parametric family of valid inequalities for $W$ based on a facet of the simple mixed integer set

$$
Q^{2}=\{v \in \mathbb{R}, y, z \in \mathbb{Z}: v+\alpha y+z \geq \beta, v, y \geq 0\} .
$$

They showed that for any $\alpha \in \mathbb{R}$ that satisfies (i) $\hat{b}>$ $\alpha>0$, and (ii) $1 / \alpha \geq\lceil\hat{b} / \alpha\rceil>\hat{b} / \alpha$, the two-step MIR inequality with parameter $\alpha$,

$$
\begin{gathered}
\sum_{j \in J} \max \left\{c_{j}, 0\right\} v_{j}+\sum_{i \in I}\left(\rho^{\alpha} \tau^{\alpha}\left\lfloor a_{i}\right\rfloor+\min \left\{\rho^{\alpha} \tau^{\alpha},\left\lfloor\hat{a}_{i} / \alpha\right\rfloor \rho^{\alpha}\right.\right. \\
\left.\left.+\hat{a}_{i}-\left\lfloor\hat{a}_{i} / \alpha\right\rfloor \alpha,\left\lceil\hat{a}_{i} / \alpha\right\rceil \rho^{\alpha}\right\}\right) x_{i} \geq \rho^{\alpha} \tau^{\alpha}\lceil b\rceil,
\end{gathered}
$$

where $\tau^{\alpha}=\lceil\hat{b} / \alpha\rceil$, and $\rho^{\alpha}=\hat{b}-\alpha\lfloor\hat{b} / \alpha\rfloor$, is valid for $W$. The two-step MIR inequality is a special case of the two-slope inequality of Gomory and Johnson (1972) (see Araoz et al. 2003) and similar to the MIR inequality; in fact, it is a rank two MIR inequality. Furthermore, the MIR inequality is just a two-step MIR inequality for a particular choice of $\alpha$.

Gomory (1969) derived the GMI cut for pure integer programs from a facet of the master cyclic group 
polyhedron (MCGP). Conversely, the MIR inequality applied to MCGP yields a facet-defining inequality. Two-step MIR inequalities are similar in that they too define facets of MCGP for appropriate choices of $\alpha$ (Dash and Günlük 2006a). Furthermore, scaled MIR inequalities (MIR inequalities applied to the defining equation of MCGP after multiplying it by an integer) are also facet defining for MCGP, as are scaled two-step MIR inequalities. Gomory et al. (2003) performed a "shooting experiment" and showed that the scaled MIR facets form an important class of facets of MCGP; see also Evans (2002). Using their approach, Dash and Günlük (2006b) showed that scaled two-step MIR facets, which are not scaled MIR facets, are also important.

MIR inequalities (including GMI cuts) are now routinely used in MIP software and are very useful in practice; see Balas et al. (1996) and Bixby et al. (2000). The similarities between MIR inequalities and two-step MIR inequalities suggest the possibility that two-step MIR inequalities could also be effective in practice. In this paper, we investigate the computational effectiveness of two-step MIR inequalities, where our measure of effectiveness is the additional integrality gap closed by them over and above MIR inequalities. To this end, we develop an effective algorithm to find violated two-step MIR inequalities which are sufficiently different from scaled MIR inequalities (this notion is formally defined in §2). Given a point $\left(v^{*}, x^{*}\right) \notin W$ that violates a two-step MIR inequality sufficiently different from scaled MIR inequalities, our algorithm finds one such inequality in $O\left(k^{2}\right)$ time, where $k$ is the number of nonzero entries in $x^{*}$. We note that the complexity does not depend on the data $(c, a, b)$ used in defining $W$, whereas the number of possible two-step MIR inequalities does.

We present extensive numerical tests and show that the two-step MIR inequalities are very useful for the multiple knapsack instances discussed in Atamtürk (2003). These instances have either one-sided bounds on variables, or the difference between the upper and lower bounds is nontrivial, and the constraint matrix is dense and has diverse coefficients. In particular, for the unbounded instances the average percentage gap closed is $78.6 \%$ versus $56.21 \%$, for GMI cuts + two-step MIR cuts versus GMI cuts alone. The additional gap closed by two-step MIR cuts is also noticeably larger than that due to adding scaled GMI cuts (also called $K$-cuts in Cornuéjols et al. 2003) based on the tableau rows, as reported by Fischetti and Saturni (2007). The two-step MIR cuts seem to be as effective as the cuts derived by Atamtürk (2003) from facets of mixed integer knapsacks for the unbounded instances in the above test set, and slightly less so for the bounded instances.
Unfortunately, for the MIP instances in MIPLIB 3.0, the additional gap closed by two-step MIR cuts is not large. However, as recently observed by Dash and Günlük (2008), this is because GMI cuts are very strong for these instances in the sense that no additional group cuts are violated once GMI cuts are added. Group cuts are all possible valid inequalties for $W$ that can be derived using MCGP. More recently, Fukasawa and Goycoolea (2007) extended this observation to show that in fact inequalities derived from single row relaxations do not improve the integrality gap by a nontrivial margin. The experiments in the previous two papers were motivated by the negative results for MIPLIB problems in this paper.

The rest of the paper is organized as follows: In §3, we study the separation problem for the two-step MIR inequalities and show that it can be solved efficiently when the parameter $\alpha$ is not allowed to be very small. In $\S 4$, we describe how we construct the base inequality for the two-step MIR inequalities and discuss other computational issues. In $\S 5$, we present computational results using several data sets including the MIPLIB problem library. We conclude the paper in $\S 6$.

\section{Preliminaries}

To compare the two-step MIR inequality and the MIR inequality, we normalize them so that they both have a right-hand side of $\lceil b\rceil$. After this normalization, inequality (2) has the form

$$
\frac{1}{\hat{b}} \sum_{j \in J} \max \left\{c_{j}, 0\right\} v_{j}+\sum_{i \in I}\left(\left\lfloor a_{i}\right\rfloor+f\left(\hat{a}_{i}\right)\right) x_{i} \geq\lceil b\rceil,
$$

where $f\left(\hat{a}_{i}\right)=(1 / \hat{b}) \min \left\{\hat{b}, \hat{a}_{i}\right\}$, and inequality (3) has the form

$$
\frac{1}{\rho^{\alpha} \tau^{\alpha}} \sum_{j \in J} \max \left\{c_{j}, 0\right\} v_{j}+\sum_{i \in I}\left(\left\lfloor a_{i}\right\rfloor+g^{\alpha}\left(\hat{a}_{i}\right)\right) x_{i} \geq\lceil b\rceil,
$$

where $\tau^{\alpha}=\lceil\hat{b} / \alpha\rceil, \rho^{\alpha}=\hat{b}-\alpha\lfloor\hat{b} / \alpha\rfloor$ and

$$
\begin{array}{r}
g^{\alpha}\left(\hat{a}_{i}\right)=\frac{1}{\rho^{\alpha} \tau^{\alpha}} \min \left\{\rho^{\alpha} \tau^{\alpha},\left\lfloor\hat{a}_{i} / \alpha\right\rfloor \rho^{\alpha}+\hat{a}_{i}\right. \\
\left.-\left\lfloor\hat{a}_{i} / \alpha\right\rfloor \alpha,\left\lceil\hat{a}_{i} / \alpha\right\rceil \rho^{\alpha}\right\} .
\end{array}
$$

The functions $f$ and $g^{\alpha}$ both take values in $[0,1]$ and are subadditive and extreme in a certain sense (Dash and Günlük 2006a). Notice that, written in this form, MIR and two-step MIR inequalities differ from each other in (1) how they (linearly) increase the coefficients of the continuous variables that have a positive coefficient in the original equation and (2) how they change the fractional part of the coefficients of integral variables. 
Note that $\hat{b}=\rho^{\alpha}+\left(\tau^{\alpha}-1\right) \alpha$, and therefore $\hat{b}-\rho^{\alpha} \tau^{\alpha}=$ $\left(\alpha-\rho^{\alpha}\right)\left(\tau^{\alpha}-1\right)>0$ as $\alpha>\rho^{\alpha}$ by definition and $\tau^{\alpha} \geq 2$ for all admissible $\alpha$. In other words, the coefficient of the continuous variables in the MIR inequality (4) is smaller than that of the two-step MIR inequality (5) and the difference is large when both $\tau$ and $\alpha-\rho^{\alpha}$ are large. For the coefficients of the integral variables, however, there is no dominance relationship as both inequalities are facet defining for the master cyclic group polyhedron (Dash and Günlük 2006b). We next give a numerical example to demonstrate how functions $f$ and $g^{\alpha}$ behave.

EXAMPLE 1. Let $\hat{b}=0.8$ and $\alpha=0.3$, implying $\rho=0.2$ and $\tau=3$. In this case the function $g^{\alpha}$ is piecewise linear with breakpoints at $\rho, \alpha, \alpha+\rho, 2 \alpha$, and $\hat{b}=2 \alpha+\rho$. The function $f$ is also piecewise linear with a single break point at $\hat{b}$. In Figure 1, we plot $g^{0.3}(s)$ and $f(s)$ for $s \in[0,1]$.

We next bound the difference between the cut coefficients of the two-step MIR inequality and the MIR inequality.

Lemma 1. Let $c \in[0,1)$ be given. Then, $1 /\lceil\hat{b} / \alpha\rceil \geq$ $\left|g^{\alpha}(c)-f(c)\right|$.

Proof. Let $t=\lceil\hat{b} / \alpha\rceil$. For all $c \in(\hat{b}, 1)$, the claim is correct as $g^{\alpha}(c)=f(c)=1$. When $c \in[0, \hat{b}]$, we have $f(c)=c / \hat{b}$ and $g^{\alpha}(c) \leq 1$. Hence,

$$
\begin{aligned}
& g^{\alpha}(c)-f(c) \\
& \quad=\frac{1}{\rho^{\alpha} t} \min \left\{\lfloor c / \alpha\rfloor \rho^{\alpha}+c-\lfloor c / \alpha\rfloor \alpha,\lceil c / \alpha\rceil \rho^{\alpha}\right\}-c / \hat{b},
\end{aligned}
$$

(a)

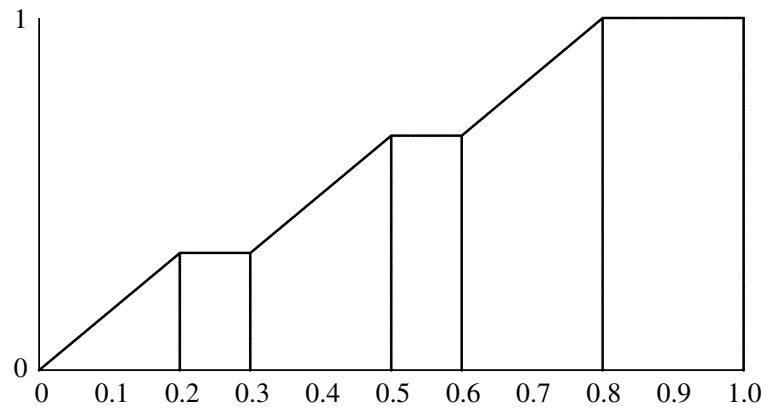

(b)

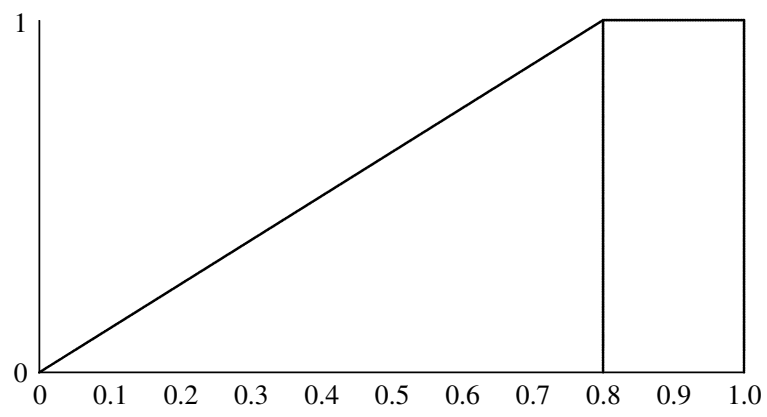

Figure 1 (a) Two-Step MIR Function $g^{0.3}(\cdot)$ and (b) MIR Function $f(\cdot)$ which is a piecewise linear function on $[0, b]$ with breakpoints at $c=(k-1) \alpha+\rho^{\alpha}$ and $c=k \alpha$ for $k=1, \ldots, t-1$. Furthermore, $g^{\alpha}(0)-f(0)=g^{\alpha}(\hat{b})-$ $f(\hat{b})=0$. Therefore, it is sufficient to consider the remaining breakpoints. If $c$ is a breakpoint, we have

$$
\begin{aligned}
g^{\alpha}(c)-f(c) & =\frac{\lceil c / \alpha\rceil}{t}-\frac{c}{\hat{b}} \\
& =\frac{\lceil c / \alpha\rceil}{t}-\frac{c / \alpha}{\hat{b} / \alpha} \leq \frac{\lceil c / \alpha\rceil}{t}-\frac{c / \alpha}{t} \leq \frac{1}{t}
\end{aligned}
$$

Similarly,

$$
\begin{aligned}
g^{\alpha}(c)-f(c) & =\frac{\lceil c / \alpha\rceil}{t}-\frac{c / \alpha}{\hat{b} / \alpha} \geq \frac{\lceil c / \alpha\rceil}{t}-\frac{\lceil c / \alpha\rceil}{t-1} \\
& =-\frac{\lceil c / \alpha\rceil}{t(t-1)} \geq-\frac{1}{t}
\end{aligned}
$$

where the last inequality follows from the fact that $\lceil c / \alpha\rceil \leq t-1$ for any breakpoint $c<\hat{b}$.

We note that this bound is tight, in the sense that for each $t \geq\lceil 1 /(1-\hat{b})\rceil$, it is possible to find an $\alpha>0$ and $c_{1}, c_{2} \in(0, \hat{b})$ such that $\lceil\hat{b} / \alpha\rceil=t$ and both $g^{\alpha}\left(c_{1}\right)-$ $f\left(c_{1}\right)$ and $f\left(c_{2}\right)-g^{\alpha}\left(c_{2}\right)$ are arbitrarily close to $1 / t$. This is achieved by setting $\alpha=(\hat{b}-\epsilon) /(t-1)$ and $c_{1}=\epsilon$, $c_{2}=\hat{b}-\epsilon$ for some small $\epsilon>0$.

We next present a simple observation based on Lemma 1. We use $\epsilon \rightarrow \delta^{+}$to denote that $\epsilon>\delta$ as $\epsilon$ tends to $\delta$.

Corollary 1. Let $c \in[0,1)$, then $\lim _{\alpha \rightarrow 0^{+}} g^{\alpha}(c)=$ $f(c)$.

In other words, if there are no continuous variables, the two-step MIR inequality converges to the MIR inequality as $\alpha$ tends to zero. If there are continuous variables, however, the MIR inequality dominates the two-step MIR inequality as $\hat{b}>\rho^{\alpha} \tau^{\alpha}$. We next show that the coefficients of the two-step MIR inequality converge to those of the MIR inequality (for both integer and continuous variables) when $\rho^{\alpha}=\hat{b}-\alpha\lfloor\hat{b} / \alpha\rfloor$ tends to $\alpha$.

Lemma 2. Let $c \in[0,1)$ and $t$ be a positive integer. Then, (i) $\lim _{\alpha \rightarrow(\hat{b} / t)}+\rho^{\alpha} \tau^{\alpha}=\hat{b}$, and (ii) $\lim _{\alpha \rightarrow(\hat{b} / t)^{+}} g^{\alpha}(c)=$ $f(c)$.

Proof. Notice that when $\alpha$ satisfies $\hat{b} /(t-1)>\alpha>$ $\hat{b} / t$, we have $[\hat{b} / \alpha\rceil=t$ and $\lfloor\hat{b} / \alpha\rfloor=t-1$. Therefore,

$$
\lim _{\alpha \rightarrow(\hat{b} / t)^{+}} \rho^{\alpha} \tau^{\alpha}=\lim _{\alpha \rightarrow(\hat{b} / t)^{+}}(\hat{b}-\alpha(t-1)) t=t \hat{b}-\hat{b}(t-1)=\hat{b} .
$$

In addition, as observed above,

$$
\lim _{\alpha \rightarrow(\hat{b} / t)^{+}} \rho^{\alpha}=\hat{b}-(\hat{b} / t)(t-1)=\hat{b} / t,
$$


and therefore,

$$
\begin{aligned}
& \lim _{\alpha \rightarrow(\hat{b} / t)^{+}}\left(\lfloor c / \alpha\rfloor \rho^{\alpha}+c-\lfloor c / \alpha\rfloor \alpha\right) \\
& =c+\lim _{\alpha \rightarrow(\hat{b} / t)^{+}}\lfloor c / \alpha\rfloor\left(\rho^{\alpha}-\alpha\right)=c .
\end{aligned}
$$

Finally, $\lim _{\alpha \rightarrow(\hat{b} / t)^{+}}\lceil c / \alpha\rceil \rho^{\alpha}=\lim _{\alpha \rightarrow(\hat{b} / t)^{+}}\lceil c / \alpha\rceil \rho^{\alpha} \geq c$. Therefore, $g^{\alpha}(c)=\min \{\hat{b}, c\}=f(c)$.

\section{Separating Two-Step MIR Inequalities}

In this section we discuss the problem of separating two-step MIR inequalities for mixed integer sets defined by a single constraint. Given a point $(\bar{v}, \bar{x}) \in$ $\mathbb{R}_{+}^{|J|+|I|}$, we define the separation problem as the problem of identifying an admissible parameter $\alpha$ that gives a most violated two-step MIR inequality (3).

For the set $W$, it is easy to show that parameter $\alpha \in \mathbb{R}$ is an admissible parameter; that is, (i) $\alpha \geq 0$, (ii) $1 / \alpha \geq\lceil\hat{b} / \alpha\rceil$, and (iii) $\lceil\hat{b} / \alpha\rceil>\hat{b} / \alpha$, if and only if $\alpha \in \mathscr{I}=\bigcup_{\tau=2}^{\infty} \mathscr{J}^{\tau}$, where

$$
g^{\tau}= \begin{cases}(\hat{b} / \tau, 1 / \tau] & \text { if } 2 \leq \tau<\lceil 1 /(1-\hat{b})\rceil, \\ (\hat{b} / \tau, \hat{b} /(\tau-1)) & \text { if } \tau \geq\lceil 1 /(1-\hat{b})\rceil .\end{cases}
$$

Notice that $g^{\tau}$ is the set of values of $\alpha$ such that $\lceil\hat{b} / \alpha\rceil=\tau$.

Our separation algorithm is based on the following properties. For $\alpha \in \mathscr{F}^{\tau}$ for a fixed $\tau$, we show that the violation of the two-step MIR inequality (right-hand side minus left-hand side) as a function of $\alpha$ (1) varies continuously with $\alpha,(2)$ is a piecewise linear function of $\alpha$, and (3) has a finite number of points (breakpoints) where the slope of the violation changes. We then conclude that the violation of a two-step MIR inequality is either maximized at one of the explicitly specified breakpoints or by taking limits as $\alpha$ approaches one of the boundaries of the interval.

We start with showing that the coefficients of the integral variables in inequality (3) change continuously as $\alpha$ changes. Remember that $\hat{c}$ denotes $c-\lfloor c\rfloor$ for $c \in \mathbb{R}$.

Lemma 3. Given $a, b \in \mathbb{R}$ and an integer $d \geq 2$, the function

$$
\begin{aligned}
& h^{a}(\alpha) \\
& =\rho^{\alpha} d\lfloor a\rfloor+\min \left\{\rho^{\alpha} d,\lfloor\hat{a} / \alpha\rfloor \rho^{\alpha}+\hat{a}-\lfloor\hat{a} / \alpha\rfloor \alpha,\lceil\hat{a} / \alpha\rceil \rho^{\alpha}\right\},
\end{aligned}
$$

where $\rho^{\alpha}=\hat{b}-\alpha(d-1)>0$, is continuous for $\alpha \in \mathscr{I}^{d}$.

Proof. Note that a function obtained by taking minimums or linear combinations of continuous functions is still continuous. Because the first term of $h^{a}(\alpha)$ is a continuous (linear) function of $\alpha$, we only need to show that the second term is also continuous. In addition, the first term in the minimization is a linear function of $\alpha$ as well. Therefore, it suffices to show that $v(\alpha)=\min \left\{\lfloor\hat{a} / \alpha\rfloor \rho^{\alpha}+\hat{a}-\lfloor\hat{a} / \alpha\rfloor \alpha,\lceil\hat{a} / \alpha\rceil \rho^{\alpha}\right\}$ is continuous.

As $\rho^{\alpha}$ is continuous and the terms in the minimization have discontinuities only when $\hat{a} / \alpha \in \mathbb{Z}$, we will only consider the case when $\alpha=\hat{a} / t$, for some $t \in \mathbb{Z}$. More precisely, we need to show that $v(\hat{a} / t)=$ $\lim _{\epsilon \rightarrow 0^{+}} v(\hat{a} / t+\epsilon)=\lim _{\epsilon \rightarrow 0^{+}} v(\hat{a} / t-\epsilon)$, First note that $v(\hat{a} / t)=t \rho^{(\hat{a} / t)}$.

In addition,

$$
\begin{aligned}
\lim _{\epsilon \rightarrow 0^{+}} v\left(\frac{\hat{a}}{t}+\epsilon\right)= & \lim _{\epsilon \rightarrow 0^{+}} \min \left\{(t-1) \rho^{(\hat{a} / t+\epsilon)}\right. \\
& \left.\quad+\hat{a}-(t-1)(\hat{a} / t+\epsilon), t \rho^{(\hat{a} / t+\epsilon)}\right\} \\
= & \min \left\{(t-1) \rho^{(\hat{a} / t)}+\hat{a} / t, t \rho^{(\hat{a} / t)}\right\}=t \rho^{(\hat{a} / t)},
\end{aligned}
$$

where the last equality follows form the fact that $\rho^{\alpha} \leq \alpha$ for all $\alpha \in \mathscr{I}^{d}$.

Finally,

$$
\begin{aligned}
& \lim _{\epsilon \rightarrow 0^{+}} v\left(\frac{\hat{a}}{t}-\epsilon\right) \\
& =\lim _{\epsilon \rightarrow 0^{+}} \min \left\{t \rho^{(\hat{a} / t-\epsilon)}+\hat{a}-t(\hat{a} / t+\epsilon),(t+1) \rho^{(\hat{a} / t-\epsilon)}\right\} \\
& \quad=\min \left\{t \rho^{(\hat{a} / t)},(t+1) \rho^{(\hat{a} / t)}\right\}=t \rho^{(\hat{a} / t)} .
\end{aligned}
$$

Therefore, in a two-step MIR inequality, the coefficients of the integral variables change without discontinuities as $\alpha \in \mathcal{I}^{d}$ changes. For a given point $(\bar{v}, \bar{x}) \in \mathbb{R}^{|J|+|I|}$ define the violation of the two-step MIR inequality with parameter $\alpha \in \mathscr{I}$ as follows:

$$
\begin{aligned}
\Delta(\alpha)=\rho^{\alpha} \tau^{\alpha}\lceil b\rceil-\sum_{j \in J} \max \left\{0, c_{j}\right\} \bar{v}_{j} \\
-\sum_{i \in I}\left(\rho^{\alpha} \tau^{\alpha}\left\lfloor a_{i}\right\rfloor+\min \left\{\rho^{\alpha} \tau^{\alpha},\left\lfloor\hat{a}_{i} / \alpha\right\rfloor \rho^{\alpha}\right.\right. \\
\left.\left.\quad+\hat{a}_{i}-\left\lfloor\hat{a}_{i} / \alpha\right\rfloor \alpha,\left\lceil\hat{a}_{i} / \alpha\right\rceil \rho^{\alpha}\right\}\right) \bar{x}_{i},
\end{aligned}
$$

where $\tau^{\alpha}=\lceil\hat{b} / \alpha\rceil$, and $\rho^{\alpha}=\hat{b}-\alpha\lfloor\hat{b} / \alpha\rfloor$ as defined earlier. Notice that if $\Delta(\alpha)>0$, then $(\bar{v}, \bar{x})$ violates the two-step MIR inequality with parameter $\alpha$. We next show that for a given point the violation of the two-step MIR inequality with parameter $\alpha$ is a continuous function of $\alpha$ and it can be maximized efficiently.

Lemma 4. Let $(\bar{v}, \bar{x}) \in \mathbb{R}_{+}^{|J|+|I|}$, be given and define $I^{\prime}=$ $\left\{i \in I: \bar{x}_{i}>0, \hat{a}_{i}<\hat{b}\right\}$. For a given $\mathcal{F}^{d}$, if $\sup _{\alpha \in \mathcal{F}^{d}}\{\Delta(\alpha)\}=$ $\Delta(\bar{\alpha})$ for some $\bar{\alpha} \in \mathcal{I}^{d}$, then one of the following statements holds:

(a) $\Delta(\bar{\alpha})=\Delta\left(\hat{a}_{i} / t\right)$ for some $i \in I^{\prime}$ and $t \in \mathbb{Z}$, or

(b) $\bar{\alpha}=1 / d$, or

(c) $\Delta(\bar{\alpha})=\Delta(\alpha)$ for all $\alpha \in \mathcal{F}^{d}$. 
Proof. Define $J^{\prime}=\left\{j \in J: \bar{v}_{j}>0, c_{j}>0\right\}$ and notice that for $\alpha \in \mathscr{I}^{d}, \tau^{\alpha}=d$. Furthermore, as $\rho^{\alpha} \tau^{\alpha} \leq$ $\min \left\{\left\lfloor\hat{a}_{i} / \alpha\right\rfloor \rho^{\alpha}+\hat{a}_{i}-\left\lfloor\hat{a}_{i} / \alpha\right\rfloor \alpha,\left\lceil\hat{a}_{i} / \alpha\right\rceil \rho^{\alpha}\right\}$ whenever $\hat{a}_{i} \geq \hat{b}$, the violation of the inequality generated by $\alpha$ can be written as

$$
\begin{aligned}
\Delta(\alpha)=\rho^{\alpha} d\lceil b\rceil-\sum_{j \in J^{\prime}} c_{j} \bar{v}_{j}-\sum_{i \in I_{0}} \rho^{\alpha} d\left\lceil a_{i}\right\rceil \bar{x}_{i} \\
-\sum_{i \in I^{\prime}}\left(\rho^{\alpha} d\left\lfloor a_{i}\right\rfloor+\left\lfloor\hat{a}_{i} / \alpha\right\rfloor \rho^{\alpha}\right. \\
\left.\quad+\min \left\{\hat{a}_{i}-\left\lfloor\hat{a}_{i} / \alpha\right\rfloor \alpha, \rho^{\alpha}\right\}\right) \bar{x}_{i},
\end{aligned}
$$

where $I_{0}=\left\{i \in I: \bar{x}_{i}>0, \hat{a}_{i} \geq \hat{b}\right\}$.

Let $L$ be the set of numbers that divide some $\hat{a}_{i}$ for $i \in I^{\prime}$. More precisely,

$$
L=\left\{\alpha \in \mathbb{R}: \alpha=\hat{a}_{i} / t \text { for some } i \in I^{\prime} \text { and some } t \in \mathbb{Z}\right\} .
$$

If $\bar{\alpha} \in L$ or $\bar{\alpha}=1 / d$, the claim is correct, so we assume that $\bar{\alpha} \notin L \cup\{1 / d\}$. Define $\alpha^{+}=\min \{\alpha \in L \cup$ $\{+\infty\}: \alpha>\bar{\alpha}\}$ and $\alpha^{-}=\max \{\alpha \in L \cup\{-\infty\}: \alpha<\bar{\alpha}\}$ so that $\alpha^{+}>\bar{\alpha}>\alpha^{-}$.

Notice that $k_{i}=\left\lfloor\hat{a}_{i} / \alpha\right\rfloor$ is constant for all $i \in I^{\prime}$ when $\alpha \in\left(\alpha^{-}, \alpha^{+}\right) \cap \mathscr{I}^{d}$, and therefore, we can define the following lower bound on $\Delta(\alpha)$ :

$$
\begin{aligned}
\hat{\Delta}(\alpha)= & \rho^{\alpha} d\lceil b\rceil-\sum_{j \in J^{\prime}} c_{j} \bar{v}_{j}-\sum_{i \in I_{0}} \rho^{\alpha} d\left\lceil a_{i}\right\rceil \bar{x}_{i} \\
& -\sum_{i \in I^{\prime}}\left(\rho^{\alpha} d\left\lfloor a_{i}\right\rfloor+k_{i} \rho^{\alpha}\right) \bar{x}_{i}-\sum_{i \in I_{1}}\left(\hat{a}_{i}-k_{i} \alpha\right) \bar{x}_{i}-\sum_{i \in I_{2}} \rho^{\alpha} \bar{x}_{i},
\end{aligned}
$$

where $I_{1}=\left\{i \in I^{\prime}: \hat{a}_{i}-k_{i} \bar{\alpha}<\bar{\rho}\right\}$ and $I_{2}=\left\{i \in I^{\prime}: \hat{a}_{i}-\right.$ $k_{i} \bar{\alpha} \geq \bar{\rho}$. Note that $\Delta(\alpha) \geq \hat{\Delta}(\alpha)$ for all $\alpha \in\left(\alpha^{-}, \alpha^{+}\right) \cap \mathscr{I}^{d}$, and $\hat{\Delta}(\bar{\alpha})=\Delta(\bar{\alpha})$.

Notice that for $\alpha \in\left(\alpha^{-}, \alpha^{+}\right) \cap \mathscr{I}^{d}$, as $\rho^{\alpha}=\hat{b}-\alpha(d-1)$, the function $\hat{\Delta}(\alpha)$ is a linear function of $\alpha$. Therefore for any $\alpha^{\prime}, \alpha^{\prime \prime} \in\left(\alpha^{-}, \alpha^{+}\right) \cap \mathscr{G}^{d}$ such that $\alpha^{\prime}<\bar{\alpha}<\alpha^{\prime \prime}$, we have

$$
\begin{aligned}
\Delta(\bar{\alpha}) & \geq \max \left\{\Delta\left(\alpha^{\prime}\right), \Delta\left(\alpha^{\prime \prime}\right)\right\} \geq \max \left\{\hat{\Delta}\left(\alpha^{\prime}\right), \hat{\Delta}\left(\alpha^{\prime \prime}\right)\right\} \\
& \geq \hat{\Delta}(\bar{\alpha}) \\
& =\Delta(\bar{\alpha}) .
\end{aligned}
$$

In other words, $\Delta\left(\alpha^{\prime}\right)=\Delta\left(\alpha^{\prime \prime}\right)=\Delta(\bar{\alpha})$, or equivalently, $\Delta(\alpha)$ is constant for $\alpha \in\left(\alpha^{-}, \alpha^{+}\right) \cap \mathcal{I}^{d}$. Therefore, the continuity of $\Delta(\alpha)$ by Lemma 3 implies that if $\alpha^{-} \in \mathscr{I}^{d}$, we have $\Delta(\bar{\alpha})=\Delta\left(\alpha^{-}\right)$. Similarly, if $\alpha^{+} \in \mathcal{I}^{d}$ we have $\Delta(\bar{\alpha})=\Delta\left(\alpha^{+}\right)$, and therefore $\Delta(\bar{\alpha})=\Delta\left(\hat{a}_{i} / t\right)$ for some $i \in I^{\prime}$ and $t \in \mathbb{Z}$, a contradiction. On the other hand, if $\alpha^{+}, \alpha^{-} \notin \mathscr{I}^{d}$ we have $\alpha \in\left(\alpha^{-}, \alpha^{+}\right) \cap \mathcal{I}^{d}=\mathscr{I}^{d}$, and $\Delta(\bar{\alpha})=\Delta(\alpha)$ for all $\alpha \in \mathscr{I}^{d}$.

Notice that Lemma 4 only analyzes the case when $\Delta^{*}=\sup _{\alpha \in \mathcal{I}^{d}}\{\Delta(\alpha)\}=\Delta(\bar{\alpha})$ for some $\bar{\alpha} \in \mathscr{I}^{d}$. As $\Delta(\alpha)$ is continuous by Lemma 3, if $\Delta^{*}>\Delta(\alpha)$ for all $\alpha \in \mathscr{I}^{d}$, then $\Delta^{*}$ must equal the limit of $\Delta(\alpha)$ as $\alpha$ approaches one of the boundary points of $\mathscr{g}^{d}$.
First consider the right boundary point and observe that

$$
\lim _{\alpha \rightarrow(\hat{b} /(t-1))^{-}} \rho^{\alpha}=\lim _{\alpha \rightarrow(\hat{b} /(t-1))^{-}} \hat{b}-\alpha\lfloor\hat{b} / \alpha\rfloor=0 .
$$

Also notice that for $(\bar{v}, \bar{x}) \geq 0$,

$$
\Delta(\alpha) \leq \rho^{\alpha} d\lceil b\rceil-\sum_{i \in I^{\prime}}\left(\rho^{\alpha} d\left\lfloor a_{i}\right\rfloor\right) \bar{x}_{i}
$$

as the remaining terms in the definition of $\Delta(\alpha)$ are nonpositive. Therefore by inequality (6) the right-hand side of inequality (7) tends to zero as $\alpha \rightarrow(\hat{b} /(t-1))^{-}$. Therefore, if $\Delta^{*}$ is attained as $\alpha$ approaches the right boundary point, there is no violated two-step MIR inequality with $\alpha \in \mathcal{F}^{d}$.

Next consider the left boundary point and note that by Lemma 2 ,

$$
\lim _{\alpha \rightarrow(\hat{b} / t)^{+}} \Delta(\alpha)=\hat{b}\lceil b\rceil-\sum_{j \in J^{\prime}} c_{j} \bar{v}_{j}-\sum_{i \in I}\left(\hat{b}\left\lfloor a_{i}\right\rfloor-\min \left\{\hat{b}, \hat{a}_{i}\right\}\right) \bar{x}_{i},
$$

which is the violation of the MIR inequality (2). Combining these observations, we conclude that if $(\bar{v}, \bar{x})$ satisfies the MIR inequality, then a violated two-step MIR inequality, if it exists, can be obtained using Lemma 4.

Also notice that for a given $\mathscr{F}^{d}$ and $i \in I$, there is at most one $t \in \mathbb{Z}_{+}$such that $\hat{a}_{i} / t \in \mathscr{I}^{d}$. Using the fact that $\mathscr{I}^{d} \subseteq(\hat{b} / d, \hat{b} /(d-1)), t$ has to satisfy $\hat{b} / d<\hat{a}_{i} / t<$ $\hat{b} /(d-1)$, implying $d / \hat{b}>t / \hat{a}_{i}>(d-1) / \hat{b}$ and therefore $t \in\left((d-1) \hat{a}_{i} / \hat{b}, d \hat{a}_{i} / \hat{b}\right)$. Clearly, only one $t \in \mathbb{Z}_{+}$can satisfy this condition. Therefore, for a given $\mathscr{G}^{d}$, and $\hat{a}_{i}$, one has to consider only $t=\left\lceil d \hat{a}_{i} / \hat{b}\right\rceil$. It is also possible that the resulting $\alpha=\hat{a}_{i} /\left\lceil d \hat{a}_{i} / \hat{b}\right\rceil$ would not be a valid parameter. Therefore, Lemma 4 leads to a linear-time separation algorithm for two-step MIR inequalities for $\alpha \in \mathscr{I}^{d}$ and we have the following result.

Lemma 5. Let $(\bar{v}, \bar{x}) \in \mathbb{R}^{|J|+|I|}$, be given and assume that $(\bar{v}, \bar{x}) \geq 0$ and it satisfies the MIR inequality. For any number $k \in \mathbb{Z}^{+}$, a most violated two-step MIR inequality can be separated in polynomial time for $\alpha \in \bigcup_{d=2}^{k} \mathscr{I}^{d}$.

Proof. Let $L^{d}$ denote the set of valid parameters for $g^{d}$ that divide some $\hat{a}_{i}$ for $i \in I^{\prime}=\left\{i \in I: \bar{x}_{i}>0\right\}$. Then we have $L^{d}=\bigcup\left\{\hat{a}_{i} /\left\lceil d \hat{a}_{i} / \hat{b}\right\rceil: i \in I^{\prime}\right\} \cap \mathcal{I}^{d}$. To find the most violated inequality for $\alpha \in \bigcup_{d=2}^{k} \mathscr{F}^{d}$, it suffices to consider $\alpha \in \bigcup_{d=2}^{k}\left(L^{d} \cup\{1 / d\}\right)$, where $\left|L^{d}\right| \leq\left|I^{\prime}\right|$. Clearly, there are at most $O(k \cdot|I|)$ candidate parameters to consider.

We would like to emphasize that Lemma 5 does not give a polynomial time separation algorithm for $\alpha \in \bigcup_{d=2}^{\infty} \mathscr{I}^{d}$. But Lemma 1 and Corollary 1 suggest that in practice one would not use very small $\alpha \mathrm{s}$, or in other words, one would not consider $\mathcal{g}^{d}$ for very large $d$. 


\section{Computational Framework}

We want to determine if two-step MIR cuts help tighten the continuous relaxations of general mixed integer programs. As MIR cuts-a subclass of two-step MIR cuts-are already known to be computationally effective, we want to measure the additional gain due to two-step MIR cuts that are sufficiently different from MIR cuts. We accordingly restrict the parameter $\alpha$ to obtain such two-step MIR cuts. In our experiments, we compare the improvement in the objective function at the root node due to MIR cuts with the improvement due to both MIR and two-step MIR cuts.

We next describe how we create base inequalities from rows of the optimal simplex tableau and from inequalities in the original formulation. We also describe how we choose the parameter $\alpha$ for two-step MIR cuts. Finally, we discuss the effect of different tableau sequences when several rounds of cuts are added.

\subsection{Transforming Base Inequalities}

Our main subroutine for generating violated MIR and two-step MIR cuts takes as input the current fractional point $\left(v^{*}, x^{*}\right)$ together with a valid base inequality

$$
\sum_{j \in J} c_{j} v_{j}+\sum_{i \in I} a_{i} x_{i} \geq b
$$

and upper and lower bounds on the variables $v$ and $x$ :

$$
l_{j} \leq v_{j} \leq u_{j} \quad \forall j \in J, \quad l_{i} \leq x_{i} \leq u_{i} \quad \forall i \in I .
$$

If we start with an equality constraint, we simply relax it to obtain the inequality form in (8). Recall that in our definition of MIR (2) and two-step MIR cuts (3), we assume all variables are nonnegative. Therefore, we first perform variable transformations to obtain a base inequality with nonnegative variables. These transformations are

$$
\begin{array}{llll}
\bar{x}_{i}=x_{i}-l_{i}, & \text { or } \quad \bar{x}_{i}=u_{i}-x_{i} & \forall i \in I, \\
\bar{v}_{j}=v_{j}-l_{j}, & \text { or } \quad \bar{v}_{j}=u_{j}-v_{j} & \forall j \in J .
\end{array}
$$

We do not generate cuts from a base inequality with unbounded variables. After these transformations, our base inequality becomes

$$
\begin{gathered}
\sum_{j \in J} c_{j}^{\prime} \bar{v}_{j}+\sum_{i \in I} a_{i}^{\prime} \bar{x}_{i} \geq b^{\prime}, \\
0 \leq \bar{v}_{j} \leq u_{j}-l_{j} \quad \forall j \in J, \quad 0 \leq \bar{x}_{i} \leq u_{i}-l_{i} \quad \forall i \in I,
\end{gathered}
$$

where $c_{j}^{\prime}, a_{i}^{\prime}$, and $b^{\prime}$ depend on the specific variable transformations performed. Dropping the upper bounds on the variables $\bar{v}_{j}$ and $\bar{x}_{i}$, we have the set $W$ in (1).

If a variable has just one bound, we use the appropriate transformation in (9). If it has both an upper bound and a lower bound, we transform the integral variables, based on the value of $\left(v^{*}, x^{*}\right)$ :

$$
\bar{x}_{i}= \begin{cases}x_{i}-l_{i} & \text { if } x_{i}^{*}<\left(l_{i}+u_{i}\right) / 2, \\ u_{i}-x_{i} & \text { if } x_{i}^{*} \geq\left(l_{i}+u_{i}\right) / 2 .\end{cases}
$$

We use the same rule for transforming the continuous variables $v_{j}$ for all $j \in J$. Note that in option (a) of the bound substitution heuristic of Marchand and Wolsey (2001), they transform continuous variables by the above rule, but not integer variables. The justification for this rule is relatively intuitive: when deriving inequalities (2) and (3), one uses the nonnegativity of the variables to relax inequality (10) to obtain an inequality with nonnegative or integral coefficients. If $\bar{v}_{j}^{*}$ and $\bar{x}_{i}^{*}$ are close to zero, this relaxation step does not increase the slack of inequality (10) too much and the relaxed inequality is more likely to yield violated inequalities.

After obtaining (10) and dropping the upper bounds on variables, we use (2) to get an MIR cut, as long as $\hat{b} \geq 10^{-5}$.

\subsection{Choosing $\alpha$ for Two-Step MIR Cuts}

After we obtain inequality (10), we try a number of different $\alpha$ values to generate violated cuts and retain only the two cuts with the highest values of violation divided by norm of cut coefficients and add them to the formulation. We consider a candidate $\alpha$ acceptable if it satisfies a number of conditions. First, Lemma 1 suggests that $\hat{b} / \alpha$ should not be too large; we restrict this number to be at most 20. (Equivalently, we require $\alpha \in \bigcup_{d=2}^{20} g^{d}$.) Corollary 1 suggests that $\alpha$ should not be too small; we insist that $\alpha \geq$ 0.0005 . Lemma 2 suggests that $\alpha$ should not be too close to $\hat{b} / t$ from above; we insist that $\alpha \geq \hat{b} / t+0.0005$.

From Lemma 5 , it suffices to try all $\hat{a}_{i} / t$ as candidates for $\alpha$, where $i \in I^{\prime}$ - the set of nonzero integral variables, $t \in \mathbb{Z}$ and $\hat{b} /\left(\hat{a}_{i} / t\right) \leq 20$, to get the most violated two-step MIR inequality. In other words, we should take the fractional parts of the coefficients in inequality (10) and divide them with small integers to obtain different $\alpha$. To reduce computation time, we choose at most one $\alpha$ for every $i \in I^{\prime}$-namely, $\hat{a}_{i} / t$, where $t$ is the smallest integer such that $\hat{a}_{i} / t$ is a valid choice for $\alpha$. In addition, we only generate cuts from sufficiently different $\alpha$. We consider two values, $\alpha_{1}$ and $\alpha_{2}$, sufficiently different if $\left|\alpha_{1}-\alpha_{2}\right| \geq$ 0.001 . Lemma 5 also suggests that one should use $1 / d$ for small integers $d$, but the resulting two-step MIR inequalities are the same as scaled MIR inequalities (Dash and Günlük 2006a), and we choose not to use them as two-step MIR inequalities.

If the starting inequality is actually an equality, we also multiply it by -1 to obtain a second base 
inequality (10). This way, we can obtain two additional two-step MIR cuts from the same base equality. Note that the MIR cuts from (10) are identical whether one first multiplies it by -1 or not. This is not true for two-step MIR cuts. For example, assume $\hat{b}=0.2$ and $\hat{a}_{i}>0.2$ for all $i \in I$. Then we cannot derive a two-step MIR cut from (10) different from the MIR cut. However, after multiplying by $-1, \hat{b}$ becomes 0.8 , and $\hat{a}_{i}<0.8$ for all $i \in I$, and we can generate several two-step MIR cuts.

\subsection{Formulation Rows}

One way to obtain base inequalities is to use rows of the original problem formulation. There are two main advantages of this approach: (1) there is little loss of precision when generating cuts from these inequalities, and (2) they are usually sparser than the simplex tableau rows. One can also aggregate several rows to obtain base inequalities (as proposed by Marchand and Wolsey 2001), but we did not implement this.

Let the current fractional point be $\left(v^{*}, x^{*}\right)$. To generate a base inequality (8), we pick a row of the problem formulation and divide it by the coefficient of an integer variable from the set $I^{\prime}=\left\{i \in I: l_{i}<x_{i}^{*}<u_{i}\right\}$. This way, from every row of the formulation, we obtain multiple base inequalities from which we can generate MIR and two-step MIR cuts. We do not use coefficients of all variables in $I^{\prime}$ in one round as this may lead to too many base inequalities. Instead, we randomly select a small number (e.g., 20\%) of them. We then transform variables as described in §4.1. Based on the resulting inequality, we generate one MIR and multiple two-step MIR cuts. Once we add the generated cuts and reoptimize, the new optimal solution leads to different base inequalities because of the use of different coefficients for divisions and different variable transformations. We call cuts generated using rows of the formulation formulation cuts.

Although the set of formulation cuts is finite, it can still be very large. In a pure integer program with $m$ rows, $n$ bounded variables, and $k$ nonzero coefficients per row, one can have $m k$ base inequalities, each of which can be transformed (via variable complementation) in $2^{k}$ possible ways giving $m k 2^{k}$ possible base inequalities. For each base inequality, there are also many different choices for $\alpha$.

\subsection{Tableau Rows}

Rows of an optimal simplex tableau are natural sources of base inequalities of the form (8) (we implicitly append slacks to inequalities in the original formulation before computing tableau rows). We use tableau rows in which the basic variable is an integer variable with a fractional value. For such a row, let $x_{1}$ stand for the basic variable. Clearly, $a_{1}=1$, and the remaining variables are at their upper or lower bounds. Therefore, after performing the variable transformation steps in $\S 4.1$, all variables other than $x_{1}$ have a value of zero. Let the right-hand side of the base inequality be $b=\lfloor b\rfloor+\hat{b}$. In this case, any admissible parameter $\alpha$ will give a violated two-step MIR inequality (3) with a left-hand side of $\rho \tau b$ and a right-hand side of $\rho \tau\lceil b\rceil$. Therefore the violation of any two-step MIR cut is simply $\rho \tau(1-\hat{b})$. Observe that for all $\alpha \in \mathscr{F}^{\tau},\lceil b\rceil$ and $\tau$ are constant, and $\rho^{\alpha}=$ $\hat{b}-\alpha(\tau-1)$, a decreasing function of $\alpha$. Therefore, using Lemma 2, maximally violated two-step MIR cuts (based on tableau rows) are simply MIR cuts.

This does not mean that two-step MIR cuts based on tableau rows are useless. Suppose base inequality (8) is derived from a row of the optimal tableau, and let $x^{\prime}$ stand for the corresponding optimal solution. After adding the MIR cut, we will obtain an optimal solution $x^{\prime \prime}$. Clearly, $x^{\prime \prime}$ satisfies the MIR cut based on inequality (8) but not necessarily all two-step MIR cuts based on inequality (8). Usually, $x^{\prime \prime}$ in fact violates some two-step MIR cuts. The above argument suggests that maximally violated two-step MIR cuts should be computed after adding violated MIR cuts and solving the strengthened linear program (LP), but without actually computing the new optimal tableau.

\subsection{Effect of Tableau Sequences}

One approach to measure the effectiveness of tableau based two-step MIR cuts would be to compare the effect of $k(\geq 1)$ rounds of MIR cuts with the effect of $k$ rounds of MIR and two-step MIR cuts combined. Here, a round of cutting means adding all cuts based on the current tableau rows. A problem with this approach is that the cuts added in one round influence the subsequent tableau and resulting base inequalities and therefore the subsequent cuts. Consider two classes of cuts $C_{1}$ and $C_{2}$ with $C_{1} \subseteq C_{2}$ (say, $C_{1}=$ MIR, $C_{2}=$ MIR + two-step MIR). In certain situations $k$ rounds of cuts from $C_{1}$ may yield a better bound than $k$ rounds of $C_{2}$.

In Figure 2, we show the behavior of five rounds of MIR tableau cuts (denoted by the solid lines) and MIR + two-step MIR tableau cuts (denoted by the dotted lines) on the MIPLIB 3.0 (Bixby et al. 1998) problems lseu and rout. We show the number of rounds on the horizontal axis and the percentage integrality gap closed on the vertical axis. For lseu, MIR + two-step MIR cuts consistently give better objective values than MIR cuts for each round of cuts. On the other hand, for rout, the gap closed by MIR cuts alone is consistently better from the second round onwards.

A starker effect of differing tableau sequences is illustrated in Figure 3. For the MIPLIB 3.0 instance fixnet6, we generate four rounds of MIR tableau cuts. We then move the first 10 rows to the end and again generate four rounds of MIR tableau cuts. In other 


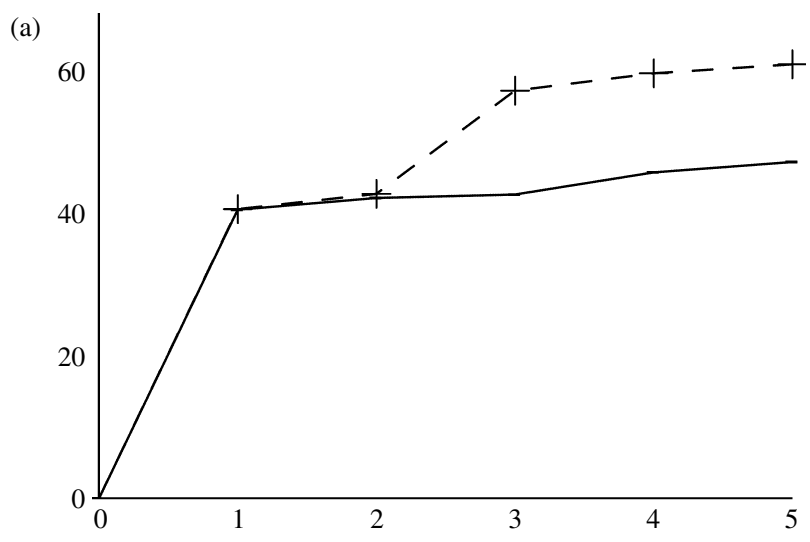

(b)

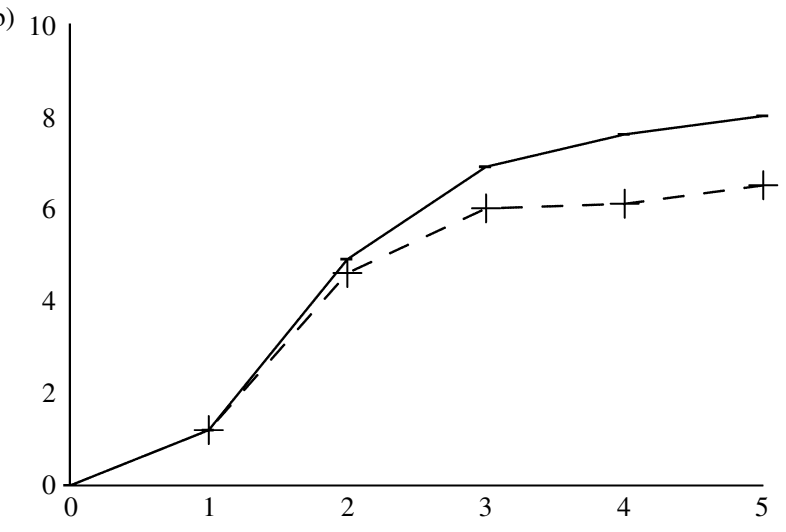

Figure 2 Gap Closed by MIR and MIR + Two-Step MIR Cuts for (a) Iseu and (b) rout

words, we apply the same procedure to the same problem instance after changing the text representations slightly. As seen in Figure 3, the gap closed after the first round is almost the same in both cases, but subsequently the gaps closed differ by up to $5 \%$.

This behavior occurs because most LP solvers terminate with a near-optimal basis-a basis for which the primal and dual solution values are feasible within given feasibility tolerances. Most practical MIP instances have numerous near-optimal bases. Different sequences of numerical operations, in this case

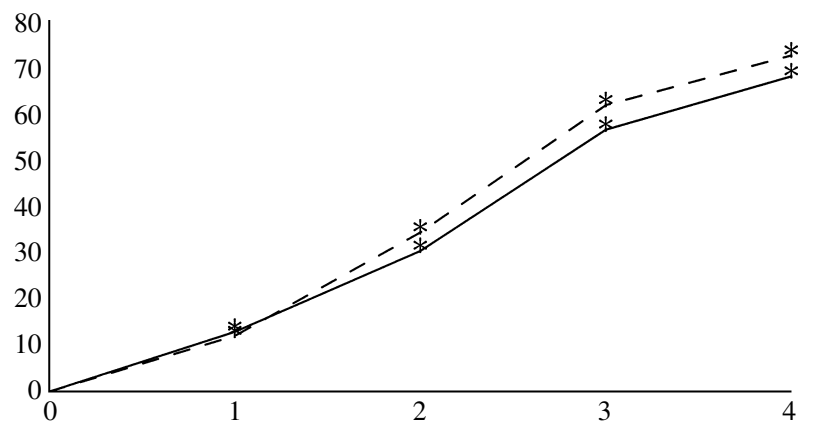

Figure 3 Effect of Different Tableau Sequences for fixnet6 triggered by the row permutations, can lead to different near-optimal bases and to different cuts.

We resolve this problem by using delayed cut generation.

\subsection{Delayed Cut Generation}

When generating multiple rounds of cuts based on tableau rows, the undesirable behavior described in $\S 4.5$ can be avoided by not adding the two-step MIR cuts until the end of MIR cut generation. Conceptually, at every round we generate the two-step MIR cuts but do not add them to the current relaxation and store them in a cut-pool. At the end, we examine the cut-pool and add violated cuts from the pool.

To implement this idea efficiently, we store the base inequalities associated with violated MIR cuts. These base inequalities are essentially the tableau rows after variable transformations. After the last round, we generate the two-step MIR cuts as discussed in $\S 4.4$ using some of the saved base inequalities. Further variable transformations are not performed at this stage. We only use base inequalities for which the associated MIR inequality has a slack of 0.7 or less. Typically, only a fraction of the generated MIR cuts satisfy this property. This fact keeps our computation cost relatively low.

To express cuts using the original variables only, we need to substitute out slack variables, including the ones associated with cuts generated in previous rounds. This can be done by saving all generated MIR cuts even if they become inactive.

\section{Numerical Results}

In the next three sections, we present numerical results obtained from several data sets. For the problem instances in these data sets, we present tables that show the effect of adding two-step MIR tableau cuts and two-step MIR formulation cuts. The first group consists of the instances randomly generated by Atamtürk (2003) to test lifted knapsack inequalities. The second group consists of four problem instances from a practical application that we encountered in steel production. The last group consists of several publicly available mixed integer test sets.

In the appendix, we show the effect of multiple rounds of cutting based on tableau rows for some selected instances. In each round we solve the (strengthened) LP relaxation to optimality and obtain the base inequalities for that round of cutting from the current simplex tableau. We display the gaps closed by MIR cuts only, and MIR and two-step MIR cuts together, for $1, \ldots, 5$ rounds. The two-step MIR cuts are generated in a delayed manner as described in $\S 4.6$. 
Dash, Goycoolea, and Günlük: Two-Step MIR Inequalities

Table 1 Atamtürk's (2003) Unbounded Instances

\begin{tabular}{|c|c|c|c|c|c|c|c|c|}
\hline \multirow[b]{2}{*}{$|/|:|J|:|R|$} & \multicolumn{3}{|c|}{ Tableau cuts } & \multicolumn{3}{|c|}{ Formulation cuts } & \multicolumn{2}{|c|}{ Scaled MIRs } \\
\hline & MIR & $+2 \mathrm{MIR}$ & No. of cuts & MIR & $+2 \mathrm{MIR}$ & No. of cuts & $1-50 \mathrm{MIR}$ & No. of cuts \\
\hline $250: 1: 50$ & 50.08 & 83.38 & 136.0 & 78.52 & 83.26 & 162.0 & 78.52 & $2,185.4$ \\
\hline $250: 1: 75$ & 54.82 & 79.16 & 203.8 & 76.12 & 79.14 & 228.4 & 76.14 & $3,014.4$ \\
\hline $250: 1: 100$ & 63.26 & 75.54 & 226.0 & 74.28 & 75.24 & 297.6 & 74.55 & $3,187.2$ \\
\hline $500: 1: 50$ & 50.68 & 80.40 & 136.0 & 76.66 & 80.26 & 176.4 & 76.67 & $2,117.2$ \\
\hline $500: 1: 75$ & 55.14 & 77.44 & 203.8 & 74.92 & 77.32 & 245.6 & 74.92 & $2,932.4$ \\
\hline $500: 1: 100$ & 63.50 & 75.96 & 269.4 & 75.12 & 75.92 & 316.2 & 75.13 & $3,293.2$ \\
\hline Average & 56.25 & 78.65 & 195.8 & 75.94 & 78.52 & 237.7 & 75.99 & $2,788.3$ \\
\hline
\end{tabular}

\subsection{Atamtürk Instances}

These instances are randomly generated mixed integer programs, with between 250 and 500 rows, between 50 and 100 integral variables, and between 1 and 20 continuous variables. The notation $|I|:|J|:|R|$ denotes a set of five instances with $|I|$ integral variables, $|J|$ continuous variables and $|R|$ rows. The above instances are divided into two groups, one with upper bounds on variables and the other without. All variables are nonnegative. The problems have the form

$$
\begin{aligned}
\max & \sum_{j \in J} h_{j} v_{j}+\sum_{i \in I} g_{i} x_{i} \\
& \sum_{j \in J} c_{r j} v_{j}+\sum_{i \in I} a_{r i} x_{i} \leq b_{r}, \quad r=1, \ldots,|R|, \\
& 0 \leq v \leq w, 0 \leq x \leq u, \quad x \in \mathbb{Z}, v \in \mathbb{R},
\end{aligned}
$$

where the upper bounds $w$ and $u$ are set to infinity for unbounded instances. These instances are available at http://ieor.berkeley.edu/ atamturk.data.
Recently, Fischetti and Saturni (2007) also performed computational tests using these instances to test the computational effectiveness of group cuts generated via interpolation. In their paper, they also present results with 1-50 scaled MIR cuts (based on the simplex tableau) and show that these simple cuts significantly reduce the integrality gap.

Tables 1 and 2 summarize our computational results with unbounded and bounded instances, respectively. In these tables, we display the percentage integrality gap closed by (1) MIR and two-step MIR cuts based on the simplex tableau, (2) MIR and two-step MIR formulation cuts, and (3) scaled MIR cuts based on the simplex tableau (taken from Fischetti and Saturni 2007). We also present the number of cuts added during the process. Cuts based on the tableau are added in one single round using the optimal simplex tableau of the initial LP relaxation. Formulation cuts, on the other hand, are added in

\begin{tabular}{|c|c|c|c|c|c|c|c|c|}
\hline \multirow[b]{2}{*}{$|/|:|J|:|R|$} & \multicolumn{3}{|c|}{ Tableau cuts } & \multicolumn{3}{|c|}{ Formulation cuts } & \multicolumn{2}{|c|}{ Scaled MIRs } \\
\hline & MIR & $+2 \mathrm{MIR}$ & No. of cuts & MIR & $+2 \mathrm{MIR}$ & No. of cuts & 1-50 MIR & No. of cuts \\
\hline $250: 5: 100$ & 61.32 & 72.34 & 256.2 & 71.16 & 72.30 & 297.60 & 71.17 & $3,196.0$ \\
\hline 250:10:50 & 50.88 & 80.02 & 136.0 & 76.32 & 79.90 & 161.60 & 76.34 & $2,100.6$ \\
\hline $250: 10: 75$ & 56.20 & 74.50 & 203.8 & 72.12 & 74.04 & 227.40 & 72.54 & $2,795.8$ \\
\hline $250: 10: 100$ & 67.40 & 75.38 & 245.4 & 74.70 & 75.38 & 295.20 & 74.69 & $2,948.4$ \\
\hline $250: 20: 50$ & 51.58 & 77.86 & 136.0 & 74.76 & 77.74 & 160.00 & 74.77 & $2,049.6$ \\
\hline $250: 20: 75$ & 61.26 & 75.62 & 202.8 & 74.36 & 75.60 & 225.20 & 74.37 & $2,586.6$ \\
\hline $250: 20: 100$ & 70.44 & 75.66 & 228.2 & 75.26 & 75.64 & 297.60 & 75.27 & $2,689.8$ \\
\hline Average & 59.87 & 75.91 & 201.2 & 74.10 & 75.80 & 237.80 & 74.16 & $2,623.8$ \\
\hline $250: 5: 50$ & 50.40 & 81.42 & 136.0 & 77.06 & 81.26 & 163.4 & & \\
\hline $250: 5: 75$ & 55.62 & 75.50 & 203.8 & 73.38 & 75.50 & 227.4 & & \\
\hline $500: 5: 50$ & 51.48 & 78.14 & 136.0 & 74.90 & 77.98 & 171.6 & & \\
\hline $500: 5: 75$ & 55.36 & 73.90 & 203.8 & 71.62 & 73.74 & 248.0 & & \\
\hline $500: 5: 100$ & 62.02 & 72.90 & 259.0 & 71.68 & 72.82 & 320.8 & & \\
\hline $500: 10: 50$ & 51.52 & 77.56 & 136.0 & 74.62 & 77.42 & 169.4 & & \\
\hline $500: 10: 75$ & 56.00 & 74.24 & 203.8 & 72.08 & 74.12 & 243.8 & & \\
\hline $500: 10: 100$ & 66.42 & 74.96 & 260.0 & 74.16 & 74.90 & 315.6 & & \\
\hline $500: 20: 50$ & 51.74 & 77.00 & 136.0 & 74.16 & 76.78 & 176.8 & & \\
\hline $500: 20: 75$ & 58.98 & 74.42 & 203.8 & 72.92 & 74.32 & 238.0 & & \\
\hline $500: 20: 100$ & 71.44 & 75.40 & 246.2 & 75.14 & 75.38 & 306.8 & & \\
\hline Average & 58.34 & 75.93 & 196.3 & 73.91 & 75.82 & 235.9 & & \\
\hline
\end{tabular}
several rounds until no more violated inequalities

Table 2 Atamtürk's (2003) Bounded Instances 
Dash, Goycoolea, and Günlük: Two-Step MIR Inequalities INFORMS Journal on Computing, Articles in Advance, pp. 1-14, () 2009 INFORMS

can be found. Each row represents the average over five instances. For example, in Table 1, the integrality gap closed by one round of MIR cuts for the five unbounded instances with $|I|=250,|J|=1$, and $|R|=$ 50 is on the average $50.08 \%$. We note that results in Fischetti and Saturni (2007) for MIR tableau cuts are consistent with our results. Finally, we divide Table 2 into two parts. In the first part, we compare our numbers with those obtained in Fischetti and Saturni (2007); there, the authors only report values for a subset of problems. The first set of averages are computed over the numbers in this part. In the second part, we give our computed values for the remaining instances, and the averages computed over all the numbers in a column.

After one round of tableau cuts, it is clear that MIR and two-step MIR cuts together close a significantly larger portion of the integrality gap than MIR cuts alone. For example, in Table 1, for $|I|:|J|:|R|=$ 250:1:75, the corresponding numbers are $54.82 \%$ and $79.16 \%$, respectively, for the tableau cuts. Note that the MIR formulation cuts are significantly stronger than the MIR tableau cuts, and therefore the difference between MIR and two-step MIR formulation cuts is less dramatic. For MIR and two-step MIR formulation cuts for this data set, we scaled the rows of the original formulation by every variable coefficient to get base inequalities.

The two-step MIR cuts also compare favorably with the scaled MIR cuts. For example, on 250:1:50, one round of MIR cuts close $50.08 \%$ of the integrality gap in our experiments (the number reported in Fischetti and Saturni 2007 is 50.07\%). Scaled MIR cuts, as reported in Fischetti and Saturni (2007), close 78.52\% of the integrality gap, whereas one round of MIR and two-step MIR cuts close $83.38 \%$ of the integrality gap, with much fewer cuts added. In our experiments we add at most four violated two-step MIR cuts per tableau or formulation row, whereas in Fischetti and Saturni (2007), the authors add all violated 1-50 scaled MIR cuts.

We note that the integrality gap closed by two-step MIR cuts is very similar for tableau and formulation cuts. This is surprising because the gap closed by MIR cuts are very different for tableau and formulation cuts. Because this behavior is not seen in other data sets, it is clearly not because of the nature of two-step MIR cuts. In addition, two-step MIR cuts based on the tableau appear to be as strong as the lifted knapsack cuts for unbounded instances (as reported in Atamtürk 2003) and weaker for the bounded instances.

We also study how the integrality gap changes when several rounds of tableau cuts are added. Our main observation is that one round of MIR and

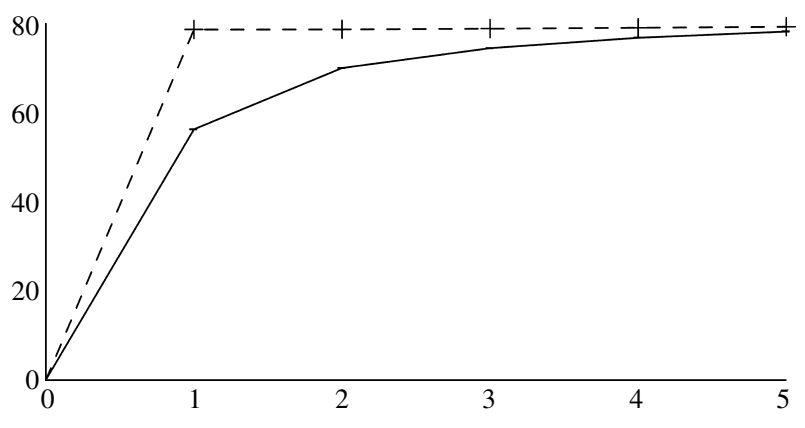

Figure 4 Multiple Rounds of MIR and Two-Step MIR Tableau Cuts

two-step MIR cuts closes the same amount of integrality gap that can be closed by many rounds of MIR cuts. In Figure 4, we show how the average integrality gap changes for unbounded Atamtürk (2003) instances (the exact numbers are given in Table A.1 in the appendix). The left axis denotes the integrality gap closed and the horizontal axis gives the number of rounds. The dashed line is for MIR and two-step MIR cuts together, and the solid line shows the MIR cuts alone. Typically, at least five rounds of MIR cuts are necessary to exceed the improvement obtained after one round of MIR and two-step MIR cuts. See Table A. 2 for similar results on the bounded Atamtürk (2003) instances.

\subsection{Steel Instances}

These are real-life mixed integer programs from the steel industry and arise from two-dimensional cutting stock problems with additional constraints. The integer programs consist of selecting the minimum "cost" set of two-dimensional patterns of rectangular plates such that the number of plates of a given order (with a given rectangular shape, and desired minimum and maximum number of plates) do not exceed the maximum for that order. There are additional constraints on usage of manufacturing resources for the selected patterns, and additional variables to count the number of completed orders. For details, see Dash et al. (2007, p. 353). We tested our two-step MIR code on four instances having 3,000 to 7,000 constraints and 4,000 to 7,000 variables.

As seen in Table 3, in all four cases, two-step MIR cuts close a relatively small but nontrivial part of

Table 3 Steel Instances

\begin{tabular}{lccccccc}
\hline & \multicolumn{3}{c}{ Tableau cuts } & & \multicolumn{3}{c}{ Formulation cuts } \\
\cline { 2 - 3 } Instance & MIR & +2 MIR & $\Delta$ & & MIR & +2MIR & $\Delta$ \\
\hline Steel 1 & 69.3 & 70.1 & 0.8 & & 50.0 & 50.9 & 0.9 \\
Steel 2 & 55.9 & 58.8 & 2.9 & & 38.9 & 47.0 & 8.1 \\
Steel 3 & 54.3 & 55.7 & 1.4 & & 46.5 & 48.1 & 1.6 \\
Steel 4 & 58.0 & 60.7 & 2.7 & 67.2 & 68.1 & 0.9 \\
Average & 59.4 & 61.3 & 1.9 & 50.65 & 53.53 & 2.88 \\
\hline
\end{tabular}


Dash, Goycoolea, and Günlük: Two-Step MIR Inequalities

the remaining integrality gap. The average remaining integrality gap closed by two-step MIR cuts is about $5 \%$ for both tableau and formulation cuts. See Table A.3 for the effect of multiple bounds of tableau cuts.

\subsection{General Mixed Integer Test Instances}

We also investigated the performance of two-step MIR cuts on the following data sets: (1) MIPLIB 3.0 (Bixby et al. 1998); (2) MIPLIB 2003 (Achterberg et al. 2005); (3) MILPlib, collected by Hans Mittelmann; and (4) a set of instances collected by Fischetti and Lodi (2003) and Fischetti et al. (2005). All of these data sets are publicly available: MIPLIB 3.0 and 2003 at http://miplib.zib.de, MILPlib at http://plato.asu. edu/sub/testcases.html, and the Fischetti and Lodi (F-L) instances at http://www.or.deis.unibo. it/research_pages/ORinstances/MIPs.html.

Table 4 lists all the problems (28/65) in MIPLIB 3.0 where two-step MIR cuts make a difference (either as tableau cuts after one round of cuts or as formulation cuts). Instances where cuts do not make any difference are indicated by an en dash. If the difference is small but nonzero, we indicate this by a " $0.00^{*}$."

In MIPLIB 3.0 instances two-step MIR cuts do not make as big a difference as in the Atamtürk (2003)

\section{Table 4 Twentyfive MIPLIB 3.0 Instances Where Two-Step MIR Cuts} Make a Difference

\begin{tabular}{|c|c|c|c|c|c|c|}
\hline \multirow[b]{2}{*}{ Instance } & \multicolumn{3}{|c|}{ Tableau cuts } & \multicolumn{3}{|c|}{ Formulation cuts } \\
\hline & MIR & $+2 \mathrm{MIR}$ & $\Delta$ & MIR & $+2 \mathrm{MIR}$ & $\Delta$ \\
\hline air04 & 6.95 & 7.42 & 0.47 & - & - & - \\
\hline air05 & 4.64 & 5.05 & 0.41 & - & - & - \\
\hline arki001 & 29.26 & 43.07 & 13.81 & 12.93 & 12.93 & - \\
\hline bell5 & 14.53 & 14.86 & 0.33 & - & - & - \\
\hline cap6000 & 41.65 & 42.27 & 0.62 & - & 12.43 & 12.43 \\
\hline dcmulti & 47.65 & 48.43 & 0.78 & - & - & - \\
\hline fast0507 & 2.00 & 2.37 & 0.37 & - & - & - \\
\hline fiber & 63.09 & 66.42 & 3.33 & 88.45 & 90.08 & 1.63 \\
\hline gen & 60.69 & 61.32 & 0.63 & 90.55 & 93.77 & 3.22 \\
\hline gesa2 & 28.53 & 28.72 & 0.19 & 68.91 & 70.29 & 1.38 \\
\hline gesa3 & 47.53 & 47.53 & - & 38.44 & 46.20 & 7.76 \\
\hline gt2 & 69.71 & 70.63 & 0.92 & 89.71 & 89.68 & -0.03 \\
\hline harp2 & 24.07 & 27.08 & 3.01 & 51.38 & 57.62 & 6.24 \\
\hline I152lav & 10.88 & 11.94 & 1.06 & 0.01 & 0.01 & - \\
\hline Iseu & 41.59 & 41.71 & 0.12 & 64.59 & 68.55 & 3.96 \\
\hline mas74 & 6.67 & 7.48 & 0.81 & - & - & - \\
\hline mas76 & 6.42 & 7.09 & 0.67 & - & - & - \\
\hline mitre & 87.21 & 90.28 & 3.07 & 94.95 & 97.65 & 2.70 \\
\hline$m k c$ & 6.83 & 7.55 & 0.72 & 4.35 & 11.87 & 7.52 \\
\hline mod008 & 20.11 & 20.35 & 0.24 & 47.38 & 63.77 & 16.39 \\
\hline p0033 & 56.82 & 57.08 & 0.26 & 56.86 & 57.65 & 0.79 \\
\hline p0282 & 3.70 & 3.70 & $0.00 *$ & 92.94 & 95.01 & 2.07 \\
\hline p0548 & 39.20 & 39.95 & 0.75 & 39.90 & 40.01 & 0.11 \\
\hline$p 2756$ & 0.54 & 0.61 & 0.07 & 0.21 & 0.21 & - \\
\hline qnet1 & 11.91 & 11.97 & 0.06 & 36.85 & 45.96 & 9.11 \\
\hline qnet1-o & 42.99 & 43.10 & 0.11 & 76.58 & 77.24 & 0.66 \\
\hline seymour & 7.69 & 7.70 & 0.01 & - & - & - \\
\hline swath & 8.42 & 26.08 & 17.66 & - & - & - \\
\hline Average & 28.26 & 30.06 & 1.80 & 34.11 & 36.82 & 2.71 \\
\hline
\end{tabular}

test set. When there is an improvement, the average additional gap closed by two-step MIR cuts is about $1.8 \%$ for tableau based cuts and about $2.7 \%$ for formulation cuts. For several instances, however, the improvement is substantial: for example, for arki001 gap closed increases from $29 \%$ to $43 \%$ with tableau cuts, and for mitre, even though formulation cuts close only $2.7 \%$ of the total integrality gap, it is more than half of the remaining gap after MIR cuts are added. Note that for $g t 2 \Delta<0$ for formulation cuts; this is because the variable transformations used to generate the base inequalites (for both MIR and two-step MIR cuts) depend on the point to be separated and different sequences of points lead to different collections of base inequalities.

In Table 5 we report on the performance of two-step MIR cuts on the remaining problem instances (MIPLIB 2003, MILPlib, and Fischetti and Lodi instances) where they make a noticeable difference either as tableau or formulation cuts. Table 5 is divided into three parts: the first part contains the problem instances from MIPLIB 2003, the second part from MILPLIB, and the last one from the Fischetti and Lodi problem set. The format of Table 5 is different from the previous tables because the optimal values of most of the instances listed in this table are not known. Instead of reporting integrality gap closed by MIR and two-step MIR cuts, we

Table 5 MIPLIB 2003/MILPlib/F-L Instances Where Two-Step MIR Cuts Make a Difference

\begin{tabular}{|c|c|c|c|c|c|c|}
\hline \multirow[b]{2}{*}{ Instance } & \multicolumn{3}{|c|}{ Tableau cuts } & \multicolumn{3}{|c|}{ Formulation cuts } \\
\hline & $\Delta \mathrm{MIR}$ & $\Delta+2 \mathrm{MIR}$ & Ratio & $\Delta \mathrm{MIR}$ & $\Delta+2 \mathrm{MIR}$ & Ratio \\
\hline atlanta-ip & 0.11 & - & 1.00 & $0.00^{*}$ & 0.01 & 3.30 \\
\hline momentum3 & 32.26 & - & 1.00 & 5.29 & 87.78 & 17.58 \\
\hline$m s c 98-i p$ & $160,260.35$ & - & 1.00 & $6,597.72$ & $2,843.32$ & 1.43 \\
\hline mzzv11 & 145.94 & 23.75 & 1.16 & - & - & - \\
\hline nsrand-ipx & 790.28 & 173.79 & 1.22 & $1,100.62$ & 1.87 & 1.00 \\
\hline protfold & 0.35 & 0.12 & 1.35 & - & - & - \\
\hline //13000 & 101.74 & 104.27 & 2.02 & 896.59 & 202.15 & 1.23 \\
\hline sp97ar & $842,872.11$ & 318.29 & 1.00 & $133,617.85$ & $65,386.22$ & 1.49 \\
\hline neos? & 219.77 & - & 1.00 & 262.16 & 48.41 & 1.18 \\
\hline neos21 & 0.36 & 0.05 & 1.14 & - & - & - \\
\hline neos3 & 302.55 & - & 1.00 & 307.32 & 106.10 & 1.35 \\
\hline nug08 & 0.61 & 2.08 & 4.42 & - & - & - \\
\hline qap10 & 0.93 & 0.07 & 1.07 & - & - & - \\
\hline $\operatorname{ran} 14 \times 18-1$ & 53.36 & 11.35 & 1.21 & - & - & - \\
\hline swath1 & 0.05 & 4.18 & 90.21 & - & - & - \\
\hline swath2 & 0.14 & 4.18 & 30.02 & - & - & 一 \\
\hline swath3 & 0.30 & 4.18 & 14.97 & - & - & - \\
\hline NSR8K & $\left({ }^{*}\right)$ & $(*)$ & $(*)$ & 53.39 & 9.48 & 1.18 \\
\hline blp-ic98 & 43.99 & 0.63 & 1.01 & 53.03 & 5.78 & 1.11 \\
\hline rail507 & 0.04 & 0.01 & 1.16 & - & - & - \\
\hline siena1 & 241.23 & - & 1.00 & $2,186.83$ & 776.07 & 1.35 \\
\hline sp97ic & $689,746.71$ & 171.82 & 1.00 & $91,361.17$ & $57,344.11$ & 1.63 \\
\hline sp98ar & $934,305.62$ & $23,128.51$ & 1.02 & $811,453.58$ & $81,201.76$ & 1.10 \\
\hline sp98ic & $313,420.27$ & 305.46 & 1.00 & $150,362.60$ & $142,333.21$ & 1.95 \\
\hline
\end{tabular}


first report the improvement in the objective function value because of MIR cuts, and then we report the additional improvement after two-step MIR cuts are added. These numbers clearly depend on how the data are scaled. To show the relative impact of the two-step MIR cuts, we next present the ratio of total improvement because of MIR and two-step MIR cuts to the improvement because of MIR cuts alone. We only report on the instances where this ratio is at least 1.1, which means that improvement as a result of two-step MIR cuts is at least $10 \%$ of the improvement as a result of MIR cuts. This is not necessarily a very good criterion (e.g., mitre of MIPLIB 3.0 does not satisfy it even though two-step MIR cuts perform well on this instance), but in the absence of optimal values, we decided to use it. When solving the LP relaxation of NSR8K strengthened with MIR cuts, we exceeded memory limits (4 GB), and we do not report on tableau cuts for this instance.

\section{Concluding Remarks}

In this paper we study how to effectively use two-step MIR inequalities as cutting planes for general mixed integer problems. Our results are in general encouraging as these cuts make a noticeable difference in some problem instances-namely, the randomly generated instances studied by Atamtürk (2003). We are, however, surprised to observe that there are a number of MIPLIB instances for which we either cannot find any violated two-step MIR inequalities once the MIR cuts are added, or cannot reduce the integrality gap by a nontrivial amount. This remains true after multiple rounds of cutting with both MIR and two-step MIR cuts; e.g., see gesa3 in Table A.4. As we have eliminated the random fluctuations in integrality gap closed because of differing tableau sequences, the observations above suggest strongly that we are unable to find violated two-step MIR cuts not because of our specific implementation choices but because two-step MIR cuts are not useful for MIPLIB instances.

This observation has been verified (to an extent) in subsequent papers motivated by the results in this paper. Dash and Günlük (2008) show for many problems that no group cuts that can be derived from the initial simplex tableau rows are violated after GMI cuts based on these rows are added. In other words, for most of the problem instances discussed in \$5.3 where we cannot find violated two-step MIR tableau cuts, there are actually no violated group cuts. Furthermore, Fukasawa and Goycoolea (2007) show that in fact no non-MIR mixed integer knapsack cuts derived from the initial tableau rows change the integrality gap by a nontrivial amount for most MIPLIB problems.

For a class of general cutting planes to be deemed effective, we feel that the additional integrality gap closed by this class when added with MIR cuts (compared to the gap closed by one round of MIR cuts alone) should be greater than the additional gap closed by a second round of MIR cuts. Two-step MIR inequalities clearly pass this test for the Atamtürk (2003) instances but fail this test for the MIPLIB instances. For example, in Table A.4, only for harp2 is it better to generate two-step MIR inequalities than to generate an additional round of MIR tableau cuts. For MIPLIB instances, it is generally better to expend computational effort to generate an additional round of MIR cuts as opposed to generating the somewhat (computationally) expensive two-step MIR cuts. However, we are convinced that this behaviour should not be construed as a weakness of two-step MIR cuts in the light of the results in Dash and Günlük (2008) and Fukasawa and Goycoolea (2007).

\section{Appendix} Table A.1 Multiple Rounds of Tableau Cuts on Atamtürk's (2003)
Unbounded Instances

\begin{tabular}{|c|c|c|c|c|c|}
\hline$|/|:|J|:|R|$ & Round 1 & Round 2 & Round 3 & Round 4 & Round 5 \\
\hline \multicolumn{6}{|l|}{ 250:1:100 } \\
\hline MIR & 63.06 & 72.2 & 72.66 & 74.78 & 75 \\
\hline$+2 \mathrm{MIR}$ & 75.24 & 75.44 & 75.72 & 75.84 & 76 \\
\hline$\Delta$ & 12.18 & 3.24 & 3.06 & 1.06 & 1 \\
\hline \multicolumn{6}{|l|}{$250: 1: 50$} \\
\hline MIR & 50.08 & 67.26 & 78.86 & 81.04 & 83.28 \\
\hline$+2 \mathrm{MIR}$ & 83.38 & 83.38 & 83.44 & 83.7 & 83.86 \\
\hline$\Delta$ & 33.3 & 16.12 & 4.58 & 2.66 & 0.58 \\
\hline \multicolumn{6}{|l|}{$250: 1: 75$} \\
\hline MIR & 54.82 & 69.44 & 73.42 & 77.46 & 79.24 \\
\hline$+2 \mathrm{MIR}$ & 79.16 & 79.16 & 79.42 & 79.64 & 79.86 \\
\hline$\Delta$ & 24.34 & 9.72 & 6 & 2.18 & 0.62 \\
\hline \multicolumn{6}{|l|}{$500: 1: 100$} \\
\hline MIR & 63.5 & 73.5 & 73.78 & 73.98 & 74.38 \\
\hline$+2 \mathrm{MIR}$ & 75.96 & 75.96 & 76.16 & 76.28 & 76.56 \\
\hline$\Delta$ & 12.46 & 2.46 & 2.38 & 2.3 & 2.18 \\
\hline \multicolumn{6}{|l|}{$500: 1: 50$} \\
\hline MIR & 50.68 & 67.74 & 77.04 & 77.6 & 81.04 \\
\hline$+2 \mathrm{MIR}$ & 80.4 & 80.4 & 80.44 & 80.94 & 81.16 \\
\hline$\Delta$ & 29.72 & 12.66 & 3.4 & 3.34 & 0.12 \\
\hline \multicolumn{6}{|l|}{$500: 1: 75$} \\
\hline MIR & 55.14 & 69.64 & 70.8 & 75.76 & 76.04 \\
\hline$+2 \mathrm{MIR}$ & 77.44 & 77.44 & 77.58 & 77.68 & 77.9 \\
\hline$\Delta$ & 22.3 & 7.8 & 6.78 & 1.92 & 1.86 \\
\hline \multicolumn{6}{|l|}{ Averages } \\
\hline MIR & 56.21 & 69.96 & 74.43 & 76.77 & 78.16 \\
\hline$+2 \mathrm{MIR}$ & 78.6 & 78.63 & 78.79 & 79.01 & 79.22 \\
\hline$\Delta$ & 22.38 & 8.67 & 4.37 & 2.24 & 1.06 \\
\hline
\end{tabular}


Dash, Goycoolea, and Günlük: Two-Step MIR Inequalities

INFORMS Journal on Computing, Articles in Advance, pp. 1-14, ( 2009 INFORMS

Table A.2 Multiple Rounds of Tableau Cuts on Atamtürk's (2003) Bounded Instances

\begin{tabular}{|c|c|c|c|c|c|}
\hline$|/|:|J|:|R|$ & Round 1 & Round 2 & Round 3 & Round 4 & Round 5 \\
\hline \multicolumn{6}{|l|}{$250: 10: 100$} \\
\hline MIR & 67.4 & 73.26 & 74.28 & 74.64 & 75.46 \\
\hline$+2 \mathrm{MIR}$ & 75.38 & 75.4 & 75.88 & 76.02 & 76.68 \\
\hline$\Delta$ & 7.98 & 2.14 & 1.6 & 1.38 & 1.22 \\
\hline \multicolumn{6}{|l|}{$250: 10: 50$} \\
\hline MIR & 50.88 & 68.16 & 74.18 & 77 & 79 \\
\hline$+2 \mathrm{MIR}$ & 80.02 & 80.02 & 80.06 & 80.64 & 80.96 \\
\hline$\Delta$ & 29.14 & 11.86 & 5.88 & 3.64 & 1.96 \\
\hline \multicolumn{6}{|l|}{$250: 10: 75$} \\
\hline MIR & 55.88 & 68.26 & 68.54 & 70.38 & 70.62 \\
\hline$+2 \mathrm{MIR}$ & 74.06 & 74.06 & 74.18 & 74.54 & 74.66 \\
\hline$\Delta$ & 18.18 & 5.8 & 5.64 & 4.16 & 4.04 \\
\hline \multicolumn{6}{|l|}{$250: 20: 100$} \\
\hline MIR & 70.44 & 74.18 & 74.9 & 75.16 & 75.42 \\
\hline$+2 \mathrm{MIR}$ & 75.66 & 75.68 & 76.06 & 76.16 & 76.4 \\
\hline$\Delta$ & 5.22 & 1.5 & 1.16 & 1 & 0.98 \\
\hline \multicolumn{6}{|l|}{$250: 20: 50$} \\
\hline MIR & 51.58 & 68.5 & 71.06 & 74.24 & 76.28 \\
\hline$+2 \mathrm{MIR}$ & 77.86 & 77.86 & 78.16 & 78.42 & 78.68 \\
\hline$\Delta$ & 26.28 & 9.36 & 7.1 & 4.18 & 2.4 \\
\hline \multicolumn{6}{|l|}{$250: 20: 75$} \\
\hline MIR & 61.26 & 71.68 & 72.66 & 73.26 & 74.06 \\
\hline$+2 \mathrm{MIR}$ & 75.62 & 75.62 & 75.8 & 76.1 & 76.68 \\
\hline$\Delta$ & 14.36 & 3.94 & 3.14 & 2.84 & 2.62 \\
\hline \multicolumn{6}{|l|}{$250: 5: 100$} \\
\hline MIR & 61.32 & 69.52 & 69.86 & 72.6 & 73.22 \\
\hline$+2 \mathrm{MIR}$ & 72.34 & 72.36 & 72.44 & 73.92 & 74.38 \\
\hline$\Delta$ & 11.02 & 2.84 & 2.58 & 1.32 & 1.16 \\
\hline \multicolumn{6}{|l|}{$250: 5: 50$} \\
\hline MIR & 50.4 & 67.68 & 76.84 & 81.56 & 82.16 \\
\hline$+2 \mathrm{MIR}$ & 81.42 & 81.42 & 81.46 & 81.86 & 82.5 \\
\hline$\Delta$ & 31.02 & 13.74 & 4.62 & 0.3 & 0.34 \\
\hline \multicolumn{6}{|l|}{$250: 5: 75$} \\
\hline MIR & 55.62 & 68.94 & 70.96 & 71.46 & 73.08 \\
\hline$+2 \mathrm{MIR}$ & 75.5 & 75.5 & 76 & 76.16 & 76.24 \\
\hline$\Delta$ & 19.88 & 6.56 & 5.04 & 4.7 & 3.16 \\
\hline \multicolumn{6}{|c|}{ 500:10:100 } \\
\hline MIR & 66.42 & 72.64 & 73.62 & 75.02 & 75.34 \\
\hline$+2 \mathrm{MIR}$ & 74.96 & 75 & 75.78 & 76.74 & 76.92 \\
\hline$\Delta$ & 8.54 & 2.36 & 2.16 & 1.72 & 1.58 \\
\hline \multicolumn{6}{|l|}{$500: 10: 50$} \\
\hline MIR & 51.52 & 68.58 & 70.34 & 75.12 & 77.36 \\
\hline$+2 \mathrm{MIR}$ & 77.56 & 77.56 & 77.7 & 77.8 & 78.22 \\
\hline$\Delta$ & 26.04 & 8.98 & 7.36 & 2.68 & 0.86 \\
\hline \multicolumn{6}{|l|}{$500: 10: 75$} \\
\hline MIR & 56 & 67.9 & 71.88 & 73.2 & 74.28 \\
\hline$+2 \mathrm{MIR}$ & 74.24 & 74.24 & 75.78 & 76.26 & 76.7 \\
\hline$\Delta$ & 18.24 & 6.34 & 3.9 & 3.06 & 2.42 \\
\hline \multicolumn{6}{|l|}{$500: 20: 100$} \\
\hline MIR & 71.44 & 74.1 & 75.04 & 75.38 & 75.68 \\
\hline$+2 \mathrm{MIR}$ & 75.4 & 75.4 & 75.92 & 76.22 & 76.5 \\
\hline$\Delta$ & 3.96 & 1.3 & 0.88 & 0.84 & 0.82 \\
\hline \multicolumn{6}{|l|}{$500: 20: 50$} \\
\hline MIR & 51.74 & 68.46 & 70.34 & 73.7 & 75.56 \\
\hline$+2 \mathrm{MIR}$ & 77 & 77 & 77.22 & 77.46 & 77.74 \\
\hline$\Delta$ & 25.26 & 8.54 & 6.88 & 3.76 & 2.18 \\
\hline \multicolumn{6}{|l|}{$500: 20: 75$} \\
\hline MIR & 58.98 & 70.62 & 71.08 & 71.26 & 71.44 \\
\hline$+2 \mathrm{MIR}$ & 74.42 & 74.42 & 74.6 & 74.7 & 74.78 \\
\hline$\Delta$ & 15.44 & 3.8 & 3.52 & 3.44 & 3.34 \\
\hline
\end{tabular}

Table A.2 (Continued)

\begin{tabular}{llcccc}
\hline$|I|:|J|:|R|$ & Round 1 & Round 2 & Round 3 & Round 4 & Round 5 \\
\hline 500:5:100 & & & & & \\
MIR & 62.02 & 69.02 & 72.58 & 73.68 & 73.96 \\
+2MIR & 72.9 & 72.92 & 74.88 & 75.6 & 76 \\
$\Delta$ & 10.88 & 3.9 & 2.3 & 1.92 & 2.04 \\
500:5:50 & & & & & \\
MIR & 51.48 & 68.52 & 69.28 & 75.02 & 80.18 \\
+2MIR & 78.14 & 78.14 & 78.28 & 78.38 & 80.54 \\
$\Delta$ & 26.66 & 9.62 & 9 & 3.36 & 0.36 \\
500:5:75 & & & & & \\
MIR & 55.36 & 67.74 & 71.34 & 72.1 & 72.36 \\
+2MIR & 73.9 & 73.9 & 75.42 & 75.8 & 75.9 \\
$\Delta$ & 18.54 & 6.16 & 4.08 & 3.7 & 3.54 \\
Averages & & & & & \\
MIR & 58.22 & 69.77 & 71.9 & 73.95 & 75.06 \\
$\quad$ +2MIR & 75.69 & 75.69 & 76.23 & 76.63 & 77.05 \\
$\Delta$ & 17.47 & 5.92 & 4.33 & 2.69 & 1.99 \\
\hline
\end{tabular}

Table A.3 Multiple Rounds of Tableau Cuts on Steel Instances

\begin{tabular}{lccccr}
\hline & \multicolumn{5}{c}{ Tableau cuts } \\
\cline { 2 - 6 } Instance & Round 1 & Round 2 & Round 3 & Round 4 & Round 5 \\
\hline Steel 1 & & & & & \\
MIR & 69.28 & 86.46 & 88.83 & 89.57 & 90.06 \\
$\quad+$ 2MIR & 70.11 & 87.08 & 88.96 & 89.63 & 90.12 \\
$\Delta$ & 0.83 & 0.62 & 0.13 & 0.06 & 0.06 \\
Steel 2 & & & & & \\
MIR & 55.86 & 68.66 & 70.41 & 71.22 & 71.57 \\
+2MIR & 58.76 & 70.76 & 71.67 & 72.46 & 72.71 \\
$\Delta$ & 2.9 & 2.1 & 1.26 & 1.24 & 1.14 \\
Steel 3 & & & & & \\
MIR & 54.31 & 66.02 & 67.66 & 68.65 & 70.81 \\
+2MIR & 55.71 & 67.44 & 69.99 & 70.61 & 72.02 \\
$\Delta$ & 1.4 & 1.42 & 2.33 & 1.96 & 1.21 \\
Steel 4 & & & & & \\
MIR & 57.99 & 70.18 & 74.66 & 76.47 & 78.23 \\
$\quad+2$ MIR & 60.65 & 72.47 & 76.74 & 78.18 & 79.47 \\
$\Delta$ & 2.66 & 2.29 & 2.08 & 1.71 & 1.24 \\
Average & & & & & \\
MIR & 59.36 & 72.83 & 75.39 & 76.48 & 77.67 \\
+2MIR & 61.31 & 74.44 & 76.84 & 77.72 & 78.58 \\
$\Delta$ & 1.95 & 1.61 & 1.45 & 1.24 & 0.91 \\
\hline
\end{tabular}

Table A.4 Multiple Rounds of Tableau Cuts on Selected MIPLIB Instances

\begin{tabular}{lccccc}
\hline Instance & Round 1 & Round 2 & Round 3 & Round 4 & Round 5 \\
\hline cap6000.mps & & & & & \\
$\quad$ MIR & 41.65 & 57.87 & 60.16 & 61.51 & 61.82 \\
$\quad+2$ MIR & 42.27 & 59.02 & 61.65 & 62.05 & 62.27 \\
$\Delta$ & 0.62 & 1.15 & 1.49 & 0.54 & 0.45 \\
dcmulti.mps & & & & & \\
$\quad$ MIR & 47.65 & 55.45 & 61.68 & 64.31 & 68.35 \\
$\quad+$ 2MIR & 48.43 & 56.08 & 62.22 & 64.58 & 68.82 \\
$\Delta$ & 0.78 & 0.63 & 0.54 & 0.27 & 0.47 \\
gesa2.mps & & & & & \\
MIR & 28.53 & 59.98 & 69.31 & 74.48 & 77.38 \\
$\quad+2$ MIR & 28.72 & 60.01 & 69.65 & 74.96 & 77.78 \\
$\Delta$ & 0.19 & 0.03 & 0.34 & 0.48 & 0.4 \\
gesa3.mps & & & & & \\
MIR & 47.53 & 50.3 & 53.78 & 56.19 & 57.9 \\
$\quad$ +2MIR & 47.53 & 50.3 & 53.78 & 56.19 & 57.92 \\
$\Delta$ & 0 & 0 & 0 & 0 & 0.02 \\
\hline
\end{tabular}


Table A.4 (Continued)

\begin{tabular}{lccccc}
\hline Instance & Round 1 & Round 2 & Round 3 & Round 4 & Round 5 \\
\hline gen.mps & & & & & \\
MIR & 60.69 & 64.61 & 65.53 & 67.64 & 68.63 \\
$\quad$ +2MIR & 61.48 & 64.65 & 65.57 & 67.76 & 68.72 \\
$\Delta$ & 0.79 & 0.04 & 0.04 & 0.12 & 0.09 \\
harp2.mps & & & & & \\
$\quad$ MIR & 24.07 & 30.16 & 31.11 & 31.9 & 31.9 \\
$\quad$ +2MIR & 27.08 & 32.5 & 32.79 & 33.01 & 33.01 \\
$\Delta$ & 3.01 & 2.34 & 1.68 & 1.11 & 1.11 \\
p0201.mps & & & & & \\
MIR & 26.71 & 39.72 & 46.98 & 51.08 & 55.25 \\
$\quad$ +2MIR & 26.71 & 40.64 & 47.3 & 52.04 & 57.16 \\
$\Delta$ & 0 & 0.92 & 0.32 & 0.96 & 1.91 \\
\hline
\end{tabular}

\section{References}

Achterberg, T., T. Kock, A. Martin. 2005. MIPLIB 2003. ZIB Technical Report 05-28, Berlin.

Aráoz, J., L. Evans, R. E. Gomory, E. L. Johnson. 2003. Cyclic group and knapsack facets. Math. Programming 96(2) 377-408.

Atamtürk, A. 2003. On the facets of the mixed-integer knapsack polyhedron. Math. Programming 98(1-3) 145-175.

Balas, E., S. Ceria, G. Cornuéjols, G. Natraj. 1996. Gomory cuts revisited. Oper. Res. Lett. 19(1) 1-9.

Bixby, R. E., S. Ceria, C. M. McZeal, M. W. P. Savelsbergh. 1998. An updated mixed integer programming library: MIPLIB 3.0. Optima 58 12-15.

Bixby, R. E., M. Fenelon, Z. Gu, E. Rothberg, R. Wunderling. 2000. MIP: Theory and practice-Closing the gap. M. J. D. Powell, S. Scholtes, eds. System Modelling and Optimization: Methods, Theory, and Applications. Kluwer Academic Publishers, Boston, 19-49.

Cornuéjols, G., Y. Li, D. Vandenbussche. 2003. K-cuts: A variation of Gomory mixed integer cuts from the LP tableau. INFORMS J. Comput. 15(4) 385-396.
Dash, S., O. Günlük. 2006a. Valid inequalities based on simple mixed-integer sets. Math. Programming 105(1) 29-53.

Dash, S., O. Günlük. 2006b. Valid inequalities based on the interpolation procedure. Math. Programming 106(1) 111-136.

Dash, S., O. Günlük. 2008. On the strength of Gomory mixed-integer cuts as group cuts. Math. Programming 115(2) 387-407.

Dash, S., J. Kalagnanam, C. Reddy, S. Song. 2007. Production design for plate products in the steel industry. IBM J. Res. Development 51(3/4) 345-362.

Evans. L. 2002. Cyclic groups and knapsack facets with applications to cutting planes. Ph.D. thesis, Georgia Institute of Technology, Atlanta.

Fischetti, M., A. Lodi. 2003. Local branching. Math. Programming Ser. B 98(1-3) 23-47.

Fischetti, M., C. Saturni. 2007. Mixed-integer cuts from cyclic groups. Math. Programming 109(1) 27-54.

Fischetti, M., F. Glover, A. Lodi. 2005. The feasibility pump. Math. Programming 104(1) 91-104.

Fukasawa, R., M. Goycoolea. 2007. On the exact separation of mixed integer knapsack cuts. M. Fischetti, D. P. Williamson, eds. Integer Programming and Combinatorial Optimization, Proc. 12th Internat. IPCO Conf. Lecture Notes in Computer Science, Vol. 4513. Springer, Berlin, 225-239.

Gomory, R. E. 1969. Some polyhedra related to combinatorial problems. Linear Algebra Appl. 2(4) 451-558.

Gomory, R. E., E. L. Johnson. 1972. Some continuous functions related to corner polyhedra, II. Math. Programming 3(1) 359-389.

Gomory, R. E., E. L. Johnson, L. Evans. 2003. Corner polyhedra and their connection with cutting planes. Math. Programming 96(2) 321-339.

Marchand, H., L. A. Wolsey. 2001. Aggregation and mixed integer rounding to solve MIPs. Oper. Res. 49(3) 363-371.

Wolsey, L. A. 1998. Integer Programming. John Wiley \& Sons, New York. 